\title{
Geometry-Induced Rigidity in Elastic Torus from Circular to Oblique Elliptic Cross-Section
}

\author{
B. H. Sun ${ }^{a, 1}$ \\ ${ }^{a}$ School of Civil Engineering \& Institute of Mechanics and Technology, Xi'an University of Architecture and Technology, Xi'an 710055, China
}

\section{ARTICLE INFO}

Keywords:

elliptic torus, oblique, nonlinear defor-

mation, Gauss curvature, Maple

\begin{abstract}
A B S T R ACT
For a given material, different shapes correspond to different rigidities. In this paper, the radii of the oblique elliptic torus are formulated, a nonlinear displacement formulation is presented and numerical simulations are carried out for circular, normal elliptic and oblique tori, respectively. Our investigation shows that both the deformation and the stress response of an elastic torus are sensitive to the radius ratio, and indicate that the analysis of a torus should be done by using the bending theory of shells rather than membrance theory. Numerical study demonstrates that the inner region of the torus is stiffer than the outer region due to the Gauss curvature. The study also shows that an elastic torus deforms in a very specific manner, as the strain and stress concentration in two very narrow regions around the top and bottom crowns. The desired rigidity can be achieved by adjusting the ratio of minor and major radii and the oblique angle.
\end{abstract}

\section{Contents}

1 Introduction

2 The principal radii of curvature of the rotated ellipse and oblique elliptic torus

3 Nonlinear displacement formulation of the oblique elliptic elastic torus

4 Numerical study of the elastic oblique elliptic torus 7 4.1 A circular torus . . . . . . . . . . . 7

4.2 A normal elliptic torus . . . . . . . . . 7

4.3 Oblique elliptic torus vs. normal elliptic torus 7

4.4 Discussions ............ . 8

5 Conclusions

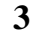

7

8

(1)

\section{Introduction}

From common sense, we know that it is easier to crack an egg on its side than at its tip, as shown in Fig.1. This kind of rigidity is an intrinsic mechanical feature of curved shells, which is called geometry-induced rigidity. Many natural and man-made objects take the shape of shells to gain better rigidity. Hence Lazarus, Florijn and Reis (2012) and Vella, Ajdari, Vaziri and Boudaoud (2012) studied the egglike shell geometry-induced rigidity of non-spherical pressurized elastic shells (ellipsoidal and cylindrical). The human foot evolution also takes advantage of curvature to increase its stiffness, hence Venkadesan, Yawar, Eng, Dias, Singh, Tommasini, Haims, Bandi and Mandre (2020) investigate the role of transverse curvature in stiffening the human foot. They considered the human foot as an elastic cantilever

\footnotetext{
*Corresponding author.

@ sunbohua@xauat.edu.cn (B.H. Sun)

을 imt.xauat. edu.cn (B.H. Sun); +8615001102877 (B.H. Sun)
}

cylindrical shell and demonstrated that, if the transverse curvature of the shell increases then stiffness of the shell increases in the longitudinal direction.

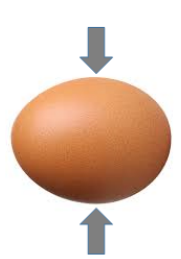

(a)

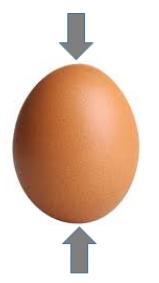

(b)
Figure 1: (a) Egg under side force (b) Egg under tip force.

All these works are about the shells that have either positive Gauss curvature (i.e., egg) or zero curvature (i.e., cylinder). However, there is another kind of shell, namely the torus (or toroidal shells), whose Gauss curvature can be positive, negative and zero as well. The Gauss curvature of the torus is shown in Fig. 2. The crowns of the torus are where the Gauss curvature of the surface changes sign.

The torus is a surface of mixed type, and little is known in general about elastic shells of mixed type. By 'mixed type', we mean that the torus is an elliptic surface in its outer half and hyperbolic in its inner half, where it looks locally like a saddle. As we shall show, an elastic torus deforms in a very specific manner, as the strain and stress concentrate in two very narrow regions, which are located around the top and bottom crowns (Audoly and Pomeau, 2010).

Doughnuts and car tubes might be two of the most common examples of real-world tori ( shown in Fig. 3). Torus structures are also often found in the water tower designs. Among regular shells, such as circular cylindrical shells, conical shells, spherical shells, and tori, the deformation of the torus is one of the most difficult topics due to its complicated topology.

The torus has been studied for more than 110 years. For 


\section{Geometry-Induced Rigidity in Elastic Torus}
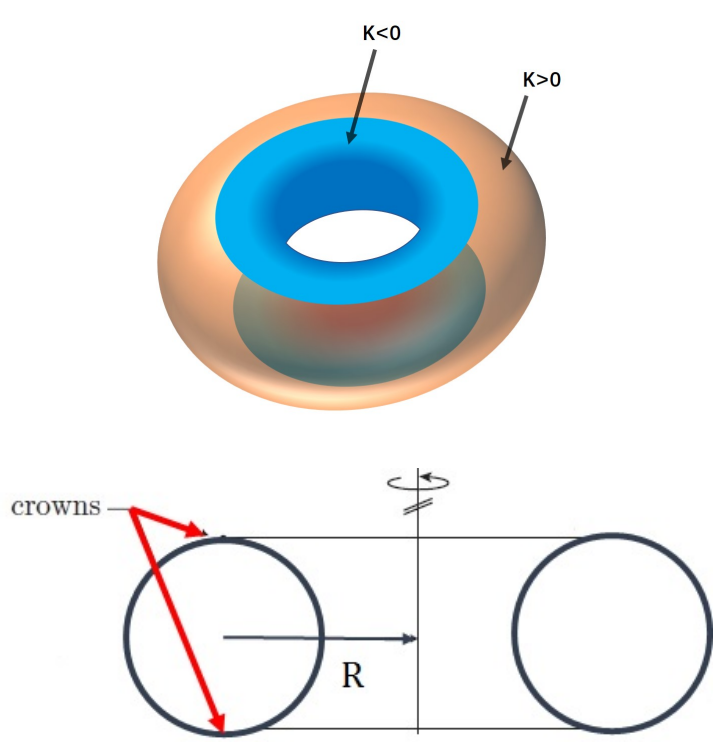

Figure 2: The sign of the Gauss curvature and crowns in a torus.

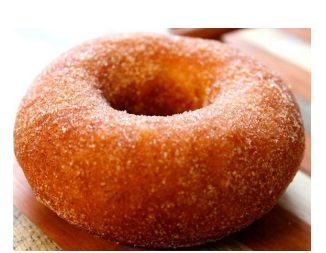

(a)

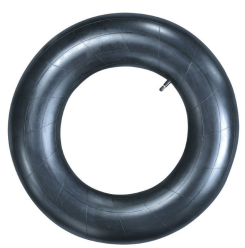

(b)
Figure 3: (a) Doughnut (b) Tube.

a review on the analysis of axisymmetric toroidal shells, including a historical perspective, we refer to the literature (Timoshenko and Woinowsky-Krieger, 1959; Kraus, 1967; Calladine, 1983; Zhang, Ren and Sun, 1990; Libai and Simmonds, 1998; Audoly and Pomeau, 2010; Sun, 2012, 2013, 2021; Zingoni, 2018).

Research of the mechanics of the torus started with the torus with a circular cross-section. The very first work on the circular torus was by L. Föppl (Föppl, 1907), who applied a linearized membrane theory to the particular case of circular toroidal shells under pressure loading. Then H. Reissner (Reissner, 1912) and E. Meissner (Meissner, 1915) derived a mixed equation to determine the elastic deformation of shells of revolution under axisymmetric loading (Flügge, 1973). E. Meissner's doctorate student Hans Wissler (Wissler, 1916) applied the Reissner-Meissner equations to toroidal shells and obtained a power series solutions. In 1944, under supervision of F. Tölke, W. Chang (later changed to W. Zhang) completed his Dr.-Ing at TU Berlin and the second part of his thesis was published in 1949 (Chang, 1949) with an asymptotic solution of Tölke's toroidal equations (Tölke, 1938) in Bessel functions. Eric Reissner (Reissner, 1949), son of Hans Reissner, carried on his father's legacy and studied the problem of pure bending of curved tubes with orthogonal ho- mogenous materials and derived a general mixed-type equation of the curved tubes. Clark and Reissner (1950) and Clark (1950) studied the toroidal or ring shell problems from the point of view of the small deflection theory of thin shells of revolution loaded symmetrically with respect to their axis. Solutions to such problems are found by applying methods of asymptotic integration of a differential equation involving a large parameter multiplied by a function that vanishes at certain points. Tao (1959) found the solution in closed form, expressed in terms of a comparatively unknown function, the Heun function. Though Tao's solution is still in the form of a power series, it has some advantages over asymptotic integrations. Steele (1965) investigated the enclosed volume and weight characteristics of toroidal pressure vessels with various cross-sectional shapes are compared. In addition to the circular and elliptic cross sections, he also considered the "modified elliptic," "circulinear," "equal-stress," and "zerohoop-stress" meridians. The circular cross section is shown to provide the minimum weight toroid. Sobel and Függe (1967) studied the stability of toroidal shells under uniform external pressure. Sanders and Liepins (1969) proposed a detailed solution for toroidal membrane under internal pressure. Their solution is remarkable as non-linear membrane effects are captured by linear boundary layer equations, thereby allowing for an approximated analytical solution. Dong (1985) proposed a new solution of Novozhilov's equation of toroidal shells. Xia and Zhang (1986) studied the general solution for thin-walled curved tubes with arbitrary loadings and various boundary conditions. Yamada, Kobayashi, Ohta and Yokota (1989) discussed the free vibration of a toroidal shell with elliptical cross-section. Zhang and Zhang (1991) obtained the complete uniformly valid solutions for toroidal shells with nonsymmetric loading corresponding to a transition point problem. Zhang and Redekop (1992) studied the surface loading of a thin-walled toroidal shell. Zhang (1994) proposed complete asymptotic expansions of four homogeneous solutions, and a particular solution of toroidal shells, based on Novozhilov's thin shell equations, which are valid for the stress and deformation of toroidal shells of circular cross section subjected to nonsymmetric loadings. Leung and Kwok (1994) and Ming, Pan and Norton (2002) studied the free vibrations of elastic circular toroidal shells. Audoly and Pomeau $(2002,2010)$ investigated the deformation of a thin elastic torus under axisymmetric surface twist loads, namely the Gol'denveizer problem (Gol'denveizer, 1961). Sun (2010) proposed a closed form solution for axisymmetric slender elastic toroidal shells when minor to major radius ratio tends to zero. Sun (2016) obtained the exact solution of Qian's equation for slender toroidal shells. Jiammeepreecha and Chucheepsakul (2017) studied the nonlinear static analysis of an underwater elastic semi-toroidal shell, and Jiammeepreecha and Chucheepsakul (2019) further investigated the nonlinear free vibration analysis of a toroidal pressure vessel under constrained volume condition. Sun (2021) obtained an exact solution of Novozhilov's equations of elastic torus in terms of Heun functions and proposed a computational algorithm of the Novozhilov equations. 
Geometry-Induced Rigidity in Elastic Torus

Concerning the cross-section of torus, most publications have focused on the circular torus, and fww non-circular cross-section tori, for instance, the elliptic torus, have been discussed. Sutcliffe (1971) conducted the stress analysis of toroidal shells of elliptical cross-section and gave the correct principal radii of elliptic torus for the first time. Bielski (1992) discussed the influence of geometrical imperfections on buckling pressure and post-buckling behavior of elastic circular and elliptic toroidal shells. Galletly and Blachut (1995) gave a heuristic study of complete toroidal shells of elliptical cross-section which were subjected to internal (rather than external) pressure. They found that buckling under internal pressure was predicted to occur with some perfect elliptical geometries. Galletly (1998) considered the elastic buckling of an elliptic toroidal shell subjected to uniform internal pressure, and confirmed that internally pressurised elliptical toroids, unlike circular toroids, may possibly buckle,depending

on the axes ratio of the elliptical cross-section. Combescure and Galletly (1999) studied the plastic buckling of complete toroidal shells of elliptical cross-section subjected to internal pressure. Kuznetsov and Levyakov (2001) studied the nonlinear pure bending of toroidal shells of arbitrary crosssection with finite element analysis. Xu and Redekop (2006) studied the natural frequencies of an orthotropic thin toroidal shell of elliptical cross-section. Zingoni, Enoma and Govender (2015) investigated a semi-elliptic toroid and proposed an approximate bending solution that may be valid in regions adjacent to the horizontal equatorial plane. Enoma and Zingoni (2020a) presented results for the state of stress and buckling of a uniformly pressurized elastic toroidal vessel of four segments, whose overall shape is similar to the normal elliptic torus. Enoma and Zingoni (2020b) investigated the buckling of an externally pressurised toroidal shell of revolution with a doubly-symmetric parabolic-ogival crosssection, where the cross-section is bound by two parabolas.

Among the different aspects of a torus, in Section \$2.2.2.4 on page 30 of Flügge's monumental treatise on the elasticity of shells (Flügge, 1973), it is remarked that the membrane theory of shells does not yield a valid solution in the following simple situation: "The shell, Fig.4, may be cut in two parts as indicated by the broken line. The meridian of each part begins and ends with a horizontal tangent. Therefore, the meridional forces acting at each edge do not have a vertical component and cannot transmit any vertical force from one half of the shell to the other. Now, when the shell is filled with gas of pressure $p$, this pressure has a downward resultant on the inner half and an upward resultant of the same magnitude on the outer half and neither part can be in equilibrium under the action of the pressure $p$ and the forces on its edges. It follows that a membrane stress system with finite values $N_{\phi}, N_{\theta}$ is not possible in this shell under this load."

The Flügger's problem of oblique torus has not been studied since. We want to ask what happens if the torus is rotated with an angle $\beta$. This paper focuses on the geometryinduced rigidity of an elastic torus and investigates the rigidity changes from a normal elliptic torus to the oblique elliptic

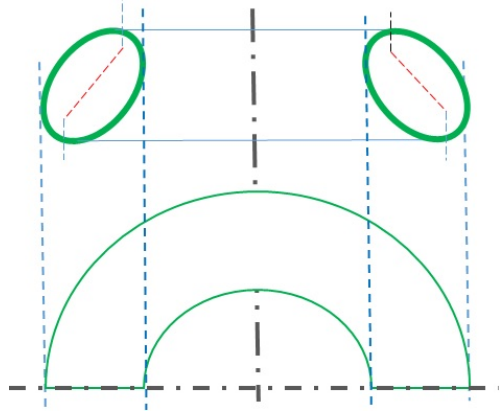

Figure 4: Flügger's problem of oblique torus in Section $\S 2.2 .2 .4$ on page 30 of W. Flügge, Stresses in Shells (Flügge, 1973).

torus ( shown in Fig. 5).

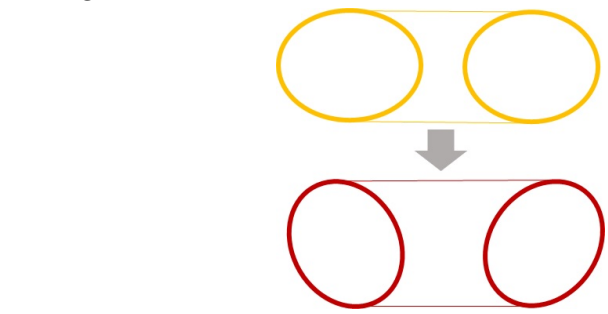

Figure 5: Geometry of torus and transformation from elliptic to oblique elliptic cross-section.

The paper is organised as follows. Section 2 formulates the principal radii of curvature of the rotated ellipse and oblique elliptic torus. Section 3 derives the nonlinear displacement governing equations of the torus. Section 4 carries out some numerical investigations on circular, normal and oblique elliptic tori, demonstrates geometry-induced rigidity of different torus and presents full discussions. Section 5 concludes with perspectives. In the appendix, a complete Maple code is provided.

\section{The principal radii of curvature of the rotated ellipse and oblique elliptic torus}

The classic Kirchhoff-Love theory of shells is well established (Love, 1920; Timoshenko and Woinowsky-Krieger, 1959; Novozhilov, 1959; Flügge, 1973). For the mechanics problem of the oblique elliptic torus, the key point is to find the principal radii of the oblique elliptic torus. Unfortunately, the radii can not be found in the literature, Therefore we first examine the geometry of the middle surfaces.

A surface of revolution is generated by the rotation of a plane curve about an axis in its plane. This generating curve is called a meridian, and an arbitrary point on the middle surface of the shell is described by specifying the particular meridian on which it is found, and by giving the value of a second coordinate which varies along the meridian and is constant on a circle around the axis of the shell, called a latitude circle (Flügge, 1973).

We identify a meridian by the angular distance $\theta$ of its 
Geometry-Induced Rigidity in Elastic Torus

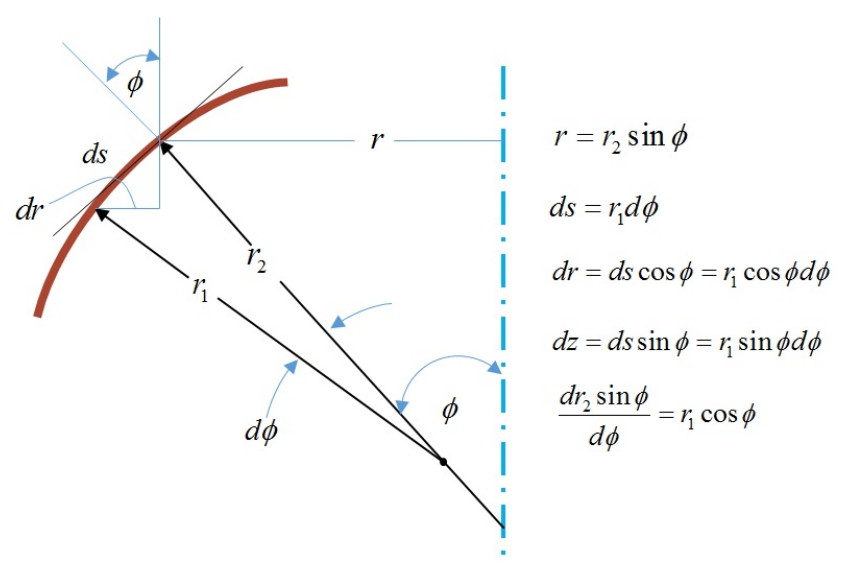

Figure 6: Surface of Revolution.

plane from that of a datum meridian and choose as second coordinate the angle $\phi$ between a normal to the shell and its axis of revolution. If the middle surface of our shell is a sphere, these coordinates are the spherical coordinates used in geography: $\theta$ is the longitude and $\phi$ is the complement to the latitude, the colatitude (Flügge, 1973).

Fig. 6 shows a meridian of the shell. Let $r$ be the distance of one of its points from the axis of rotation and $r_{1}$ its radius of curvature. In our equations we also need the length $r_{2}$, measured on a normal to the meridian between its intersection with the axis of rotation and the middle surface. It is the second radius of curvature of the shell, and we read from Fig. 6 the relation: $r=r_{2} \sin \phi$. For the line element $d s$ of the meridian we have $d s=r_{1} d \phi$, and since $d r=d s \cos \phi$ and $d z=d s \sin \phi$, we have the relations

$$
\frac{d r}{d \phi}=r_{1} \cos \phi, \quad \frac{d z}{d \phi}=r_{1} \sin \phi
$$

and Gauss-Codazzi relation

$$
\frac{d r_{2} \sin \phi}{d \phi}=r_{1} \cos \phi
$$

For the shells of revolution shown in Fig. 6, the positions of points on the middle surface will be determined by the angles $\theta$ and $\phi$. Further, let $r_{1}$ be the radius of curvature of the meridian and $r_{2}$ the radius of curvature of the normal section, tangential to the parallel circle. This second radius is equal to the segment of the perpendicular to the middle surface between this surface and the axis of the torus.

The oblique elliptic torus can be viewed as a normal elliptic torus with rotation $\beta$ around its center; the rotation takes place within the plane of cross-section of the torus. It is clear that the cross-section of the rotated torus is still elliptic. The difference between the oblique torus and normal torus is that both major and minor axes are rotated by the angle $\beta$ as shown in Fig.7.

In the following, we formulate the principal radii formulation of oblique torus. Before doing that, let's recall the formulation of the principal radii of normal elliptic torus as shown in Fig. 8, whose equation is given by $\left(\frac{x-R}{a}\right)^{2}+\left(\frac{z}{b}\right) 2=$
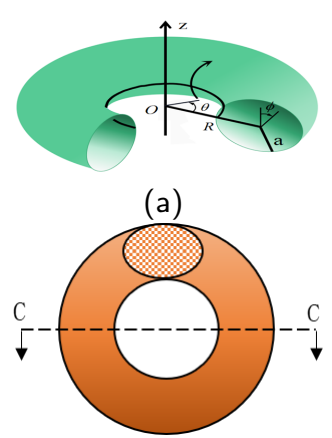

(b)

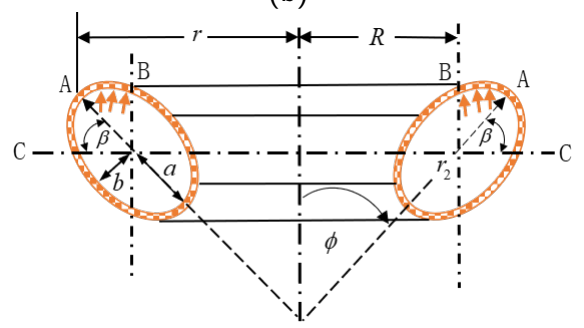

(c)

Figure 7: Geometry of the torus, the Lamé coefficients are $A_{1}=r_{1}, A_{2}=r_{2} \sin \phi$.

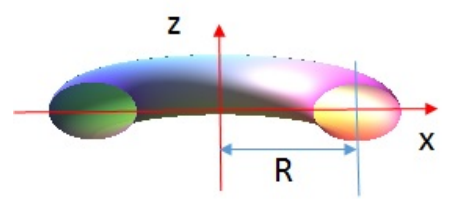

Figure 8: Geometry of the normal ellipse that is generated by rotating the curve $\left(\frac{x-R}{a}\right)^{2}+\left(\frac{z}{b}\right)^{2}=1$.

1. It leads to $z= \pm \frac{b}{a} \sqrt{a^{2}-x^{2}}$. The magnitude of the derivatives of this expression are: $\frac{d z}{d x}=\frac{b x}{a \sqrt{a^{2}-(x-R)^{2}}}=$ $\frac{b^{2}(R-x)}{a^{2} y}$ and $\frac{d^{2} z}{d x^{2}}=\frac{b^{4}}{a^{2} y^{3}}$. Referring to the Fig.6, we have $\tan \phi=\frac{d z}{d x}=\frac{R-x}{\sqrt{r_{2}^{2}-(x-R)^{2}}}$, solving it we can find the cartesian coordinates $x, y$ expressed in the meridian angle $\phi$ :

$$
\begin{aligned}
& x=R+\frac{a^{2} \sin \phi}{\sqrt{a^{2} \cos ^{2} \phi+b^{2} \sin ^{2} \phi}}, \\
& z=\frac{b^{2} \cos \phi}{\sqrt{a^{2} \cos ^{2} \phi+b^{2} \sin ^{2} \phi}} .
\end{aligned}
$$

It can be considered as the parametrization of the ellipse equation $\left(\frac{x-R}{a}\right)^{2}+\left(\frac{z}{b}\right)^{2}=1$ by the meridian angle $\phi$.

The principal radii $r_{2}$ is given by $r_{2}=\frac{x}{\sin \phi}$. Applying the Gauss-Codazzi relation $r_{1} \cos \phi=\frac{d r_{2} \sin \phi}{d \phi}$, we find principal radii $r_{1}=\frac{1}{\cos \phi} \frac{d r_{2} \sin \phi}{d \phi}$. Therefore we have the principal radii 
Geometry-Induced Rigidity in Elastic Torus

of normal elliptic surface as follows

$$
\begin{aligned}
& r_{1}=\frac{a^{2} b^{2}}{\left(a^{2} \cos ^{2} \phi+b^{2} \sin ^{2} \phi\right)^{3 / 2}}, \\
& r_{2}=\frac{R}{\sin \phi}+\frac{a^{2}}{\left(a^{2} \cos ^{2} \phi+b^{2} \sin ^{2} \phi\right)^{1 / 2}} .
\end{aligned}
$$

These relations were given by Sutcliffe (1971), and can also be found in Zingoni (2018). When the major radius $R=0$, the above radii reduced to the normal ellipsoid's radii, which can be seen in Reddy's book (Reddy, 2007).

Let us consider a rotated ellipse as shown in Fig.9.

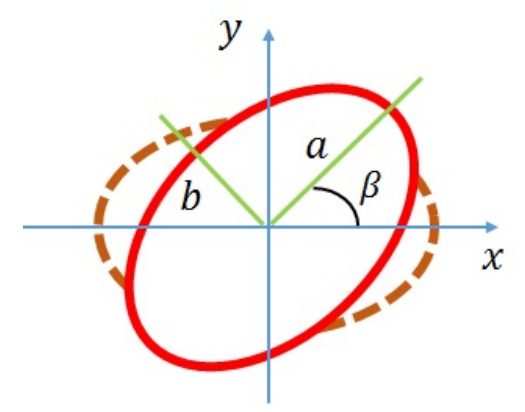

Figure 9: Geometry of the rotated ellipse: $\left(\frac{x^{\prime}-R}{a}\right)^{2}+\left(\frac{y^{\prime}}{b}\right)^{2}=1$

The point $(x, y)$ is rotated to $\left(x^{\prime}, y^{\prime}\right)$ by angle $\beta$, the coordinates transformation in between are: $x^{\prime}-R=x \cos (\beta)+$ $y \sin (\beta)$, and $y^{\prime}=-x \sin (\beta)+y \cos (\beta)$. Thus we can write the equation of the rotated ellipse in the coordinates $(x, y)$ as follows

$$
\left(\frac{x^{\prime}-R}{a}\right)^{2}+\left(\frac{z^{\prime}}{b}\right)^{2}=1 .
$$

Taking into account of relations in Eq.3, we have the position vector $\boldsymbol{r}(\phi, \theta)$ parametrization of the oblique elliptic torus

$$
\begin{aligned}
\boldsymbol{r} & =\boldsymbol{i}\left(R+\frac{a^{2} \sin \phi \cos \beta+b^{2} \cos \phi \sin \beta}{\left(a^{2} \cos ^{2} \phi+b^{2} \sin ^{2} \phi\right)^{1 / 2}}\right) \cos \theta \\
& +\boldsymbol{j}\left(R+\frac{a^{2} \sin \phi \cos \beta+b^{2} \cos \phi \sin \beta}{\left(a^{2} \cos ^{2} \phi+b^{2} \sin ^{2} \phi\right)^{1 / 2}}\right) \sin \theta \\
& +\boldsymbol{k} \frac{-a^{2} \sin \phi \sin \beta+b^{2} \cos \phi \cos \beta}{\left(a^{2} \cos ^{2} \phi+b^{2} \sin ^{2} \phi\right)^{1 / 2}} .
\end{aligned}
$$

With the position vector $\boldsymbol{r}(\phi, \theta)$, we can draw torus as shown in Fig. 10

Using the position vector in Eq. 6, we can calculate the first fundamental form $I=E d \phi^{2}+2 F d \phi d \theta+G d \theta^{2}$, where $E=\boldsymbol{r}_{, \phi} \cdot \boldsymbol{r}_{, \phi}=A_{\phi}^{2}, F=\boldsymbol{r}_{, \phi} \cdot \boldsymbol{r}_{, \theta}=0$ and $G=\boldsymbol{r}_{, \theta} \cdot \boldsymbol{r}_{, \theta}=$ $A_{\theta}^{2}$, and the Láme coefficients $A_{\phi}=\frac{a^{2} b^{2}}{\left(a^{2} \cos ^{2} \phi+b^{2} \sin ^{2} \phi\right)^{3 / 2}}$ and $A_{\theta}=R+\frac{a^{2} \sin \phi \cos \beta+b^{2} \cos \phi \sin \beta}{\left(a^{2} \cos ^{2} \phi+b^{2} \sin ^{2} \phi\right)^{1 / 2}}$.

The second fundamental form $I I=L d \phi^{2}+2 M d \phi d \theta+$ $N d \theta^{2}$, where $L=A_{\phi}^{2}, M=0$ and $N$ is not presented here due to its length.

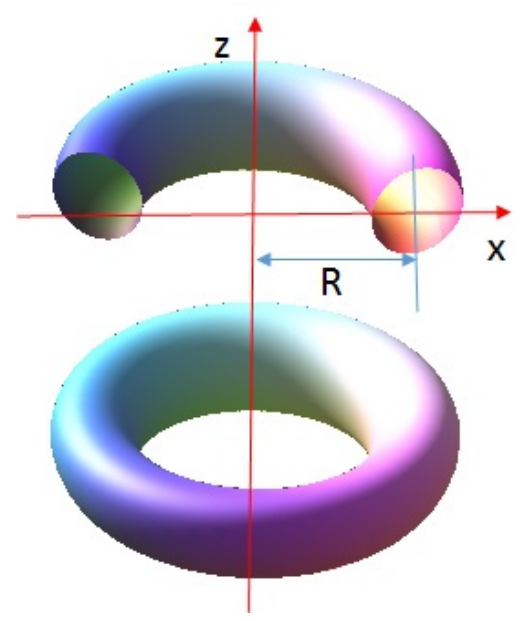

Figure 10: Oblique elliptic torus is plotted by the Eq.6.

Hence we obtain the principle radii of the oblique elliptic torus $r_{1}=\frac{E}{L}$ and $r_{2}=\frac{G}{N}$, namely

$$
r_{1}=\frac{a^{2} b^{2}}{\left(a^{2} \sin ^{2} \phi+b^{2} \cos ^{2} \phi\right)^{3 / 2}},
$$$$
r_{2}=\frac{1}{\sin (\phi+\beta)}\left[R+\frac{a^{2} \sin \phi \cos \beta+b^{2} \cos \phi \sin \beta}{\left(a^{2} \sin ^{2} \phi+b^{2} \cos ^{2} \phi\right)^{1 / 2}}\right] \text {. }
$$

The derived principal radii reveal that the meridian radii $r_{1}$ has nothing to do with the rotation angle $\beta$ while $r_{2}$ is strongly affected by the rotation $\beta$. For a better imagination, an example of $r_{2}$ is shown in Fig.11.

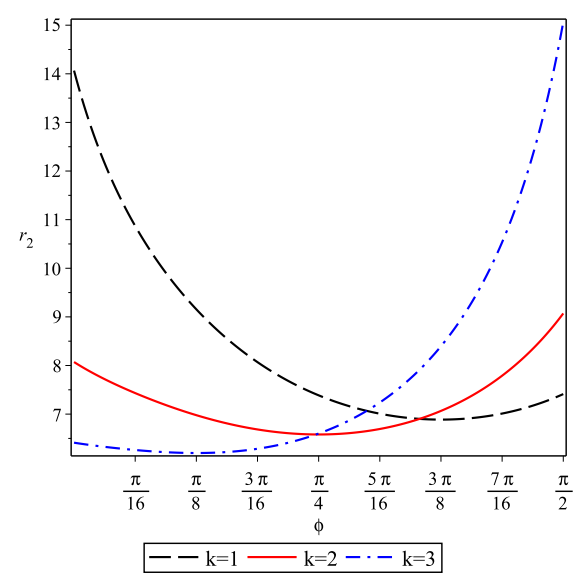

Figure 11: The principle radii $r_{2}$ vs. the oblique angle $\beta$. The draw is plotted with data: $a=2, b=1, R=5$ and $\beta=\frac{\pi}{8} k$, and $k=1,2,3$.

The geometrical interpretation of Eq.7 can be understood as follows: Tilting the elliptic cross-section by an angle $\beta$ is not expected to change the local curvature (value of $r_{1}$ ) at any given point, because applying a rigid rotation to a curve does not change the shape of the curve. It is only the second radius $r_{2}$ that is expected to depend on $\beta$. According to the invariance principal of arc-length in a rotating coordinate system, for the rotated elliptic torus $d s_{1}=r_{1} d \phi=$ 


\section{Geometry-Induced Rigidity in Elastic Torus}

$\frac{a^{2} b^{2}}{\left(a^{2} \sin ^{2} \phi+b^{2} \cos ^{2} \phi\right)^{3 / 2}} d \phi$, hence $r_{1}=\frac{a^{2} b^{2}}{\left(a^{2} \sin ^{2} \phi+b^{2} \cos ^{2} \phi\right)^{3 / 2}} ;$ and since $x^{\prime}=R+\frac{a^{2} \sin \phi \cos \beta+b^{2} \cos \phi \sin \beta}{\left(a^{2} \cos ^{2} \phi+b^{2} \sin ^{2} \phi\right)^{1 / 2}}$, thus $d s_{2}=r_{2} d \theta=$ $\frac{x^{\prime}}{\sin (\phi+\beta)} d \theta=\frac{1}{\sin (\phi+\beta)}\left[R+\frac{a^{2} \sin \phi \cos \beta+b^{2} \cos \phi \sin \beta}{\left(a^{2} \cos ^{2} \phi+b^{2} \sin ^{2} \phi\right)^{1 / 2}}\right] d \theta$, therefore $r_{2}=\frac{1}{\sin (\phi+\beta)}\left[R+\frac{a^{2} \sin \phi \cos \beta+b^{2} \cos \phi \sin \beta}{\left(a^{2} \cos ^{2} \phi+b^{2} \sin ^{2} \phi\right)^{1 / 2}}\right]$.

\section{Nonlinear displacement formulation of the oblique elliptic elastic torus}

When the linear problem was first studied, high-order and complicated governing equations of a torus under symmetric loads were reduced to lower-order, ordinary differential equations (ODE) by Hans Reissner (Reissner, 1912). His colleague at ETH, Meissner (Meissner, 1915) derived a complex-form equation for the shell of revolution. Hence, the first complex-form equation of the shells of revolution including tori is called the Reissner-Meissner equation, which is an ODE system for the shear force $Q$ and the rotation $\chi$ (Flügge, 1973). In 1959, Novozhilov (Novozhilov, 1959) published his celebrated monograph on the complex-form theory of shells and formulated symmetrical deformation of a torus with a circular cross-section.

Regarding the formulation of the elastic torus, the governing equations that all publications adopted are either the Reissner-Meissner's mixed formulation or Novozhilov's complexform one. Although those formulations have some advantages, they cannot be used for vibration and nonlinear problems. Therefore, it is desirable to have nonlinear displacement formulations for the elastic torus.

Concerning the displacement formulation of an elastic torus, Sun (2010) derived linear displacement-type equations and obtained a closed-form solution for the special case when radius ratio $\frac{a}{R} \rightarrow 0$. However, this special solution cannot capture the full mechanics nature of the torus and therefore are needed to be improved further. This paper investigates the mechanical response of the oblique elliptic torus for arbitrary ratio $\frac{a}{R}$.

Regarding the forces shown in Fig.12, the balance equations (Reddy, 2007) are

$$
\begin{gathered}
\frac{d}{d \phi}\left(r N_{\phi}\right)-N_{\theta} r_{1} \cos \phi+r Q_{\phi}-r N_{\phi} \vartheta+r_{1} r f_{\phi}=0 \\
\frac{d}{d \phi}\left(r Q_{\phi}\right)-r_{1} r\left(\frac{N_{\phi}}{r_{1}}+\frac{N_{\theta}}{r_{2}}\right)-\frac{d}{d \phi}\left(r N_{\phi} \vartheta\right)+r_{1} r f_{\zeta}=0,
\end{gathered}
$$

where $r=r_{2} \sin \phi$, distributed loads $f_{\phi}, f_{\zeta}$ along $\phi, \zeta$ direction, and shear force

$$
Q_{\phi}=\frac{1}{r_{1} r} \frac{d}{d \phi}\left(r M_{\phi}\right)-\frac{1}{r} \cos \phi M_{\theta},
$$

where the resultant membranae forces

$$
N_{\phi}=K\left(\varepsilon_{\phi}+v \varepsilon_{\theta}\right), \quad N_{\theta}=K\left(v \varepsilon_{\phi}+\varepsilon_{\theta}\right),
$$

the resultant bending moments

$$
M_{\phi}=B\left(\kappa_{\phi}+v \kappa_{\theta}\right), \quad M_{\theta}=B\left(v \kappa_{\phi}+\kappa_{\theta}\right),
$$

where the membrane stiffness $K=\frac{E h}{1-v^{2}}$, the bending stiffness $B=\frac{E h^{3}}{12\left(1-v^{2}\right)}$, the thickness $h$, the Young modulus $E$ and the Poisson ratio $v$.

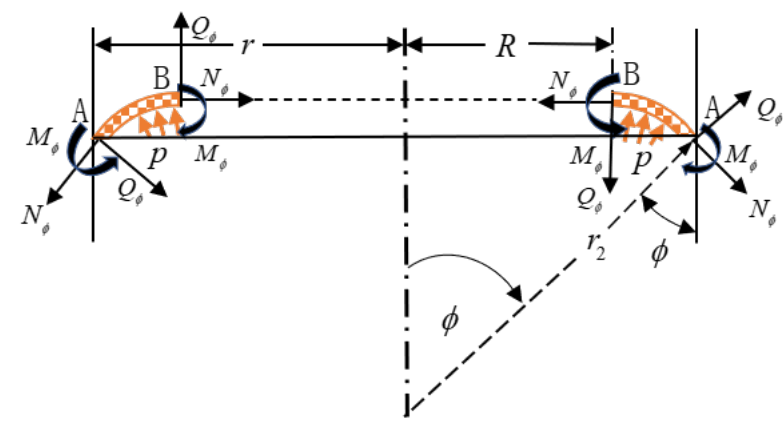

Figure 12: Loading, forces and moments of the torus

The membrane strains are

$$
\varepsilon_{\phi}=\frac{1}{r_{1}}\left(\frac{d u}{d \phi}+w\right)+\frac{1}{2} \vartheta^{2}, \quad \varepsilon_{\theta}=\frac{1}{r_{2}}(u \cot \phi+w),
$$

the change of curvature are

$$
\kappa_{\phi}=\frac{1}{r_{1}} \frac{d \vartheta}{d \phi}+\frac{1}{r_{1}^{2}} \vartheta^{2}, \quad \kappa_{\theta}=\frac{\cot \phi}{r_{2}} \vartheta
$$

and the total rotation

$$
\vartheta=\frac{1}{r_{1}}\left(u-\frac{d w}{d \phi}\right) .
$$

With the above strains and curvature change, the resultant membrane force and bending moments can be expressed in terms of displacements $u, w$ as follows:

$$
\begin{aligned}
& N_{\phi}=K\left[\frac{1}{r_{1}}\left(\frac{d u}{d \phi}+w\right)+\frac{1}{2} \vartheta^{2}+v \frac{1}{r_{2}}(u \cot \phi+w)\right], \\
& N_{\theta}=K\left[\frac{1}{r_{2}}(u \cot \phi+w)+v \frac{1}{r_{1}}\left[\left(\frac{d u}{d \phi}+w\right)+\frac{1}{2} \vartheta^{2}\right]\right], \\
& M_{\phi}=B\left[\frac{1}{r_{1}} \frac{d \vartheta}{d \phi}+\frac{1}{r_{1}^{2}} \vartheta^{2}+v \frac{\cot \phi}{r_{2}} \vartheta\right], \\
& M_{\theta}=B\left[v\left[\frac{1}{r_{1}} \frac{d \vartheta}{d \phi}+\frac{1}{r_{1}^{2}} \vartheta^{2}\right]+\frac{\cot \phi}{r_{2}} \vartheta\right] .
\end{aligned}
$$

Substituting the shear force $Q_{\phi}$ in Eq.9 into Eq.8 and produce

$$
\begin{aligned}
& \frac{d}{d \phi}\left(r N_{\phi}\right)-N_{\theta} r_{1} \cos \phi+\frac{1}{r_{1}}\left[\frac{d}{d \phi}\left(r M_{\phi}\right)\right. \\
& \left.-r \cos \phi M_{\theta}\right]-r N_{\phi} \vartheta+r_{1} r f_{\phi}=0, \\
& \frac{d}{d \phi}\left[\frac{1}{r} \frac{d}{d \phi}\left(r M_{\phi}\right)-\cos \phi M_{\theta}\right]-r_{1} r\left(\frac{N_{\phi}}{r_{1}}+\frac{N_{\theta}}{r_{2}}\right) \\
& -\frac{d}{d \phi}\left(r N_{\phi} \vartheta\right)+r_{1} r f_{\zeta}=0 .
\end{aligned}
$$

The Eq. 16 can be further simplified by substituting the constitutive relations into Eq.16, which will generate a fi- 
Geometry-Induced Rigidity in Elastic Torus

nal equations as a six order nonlinear ordinary differential equation system about displacement $u(\phi)$ and $w(\phi)$.

$$
\begin{aligned}
& K \frac{d}{d \phi}\left(r\left(\left[\frac{1}{r_{1}}\left(\frac{d u}{d \phi}+w\right)+\frac{1}{2} \vartheta^{2}+v \frac{1}{r_{2}}(u \cot \phi+w)\right]\right)\right) \\
& -K r_{1} \cos \phi\left[\frac{1}{r_{2}}(u \cot \phi+w)+v \frac{1}{r_{1}}\left[\left(\frac{d u}{d \phi}+w\right)+\frac{1}{2} \vartheta^{2}\right]\right] \\
& +B \frac{1}{r_{1}} \frac{d}{d \phi}\left(r \left[\frac{1}{r_{1}} \frac{d}{d \phi}\left[\frac{1}{r_{1}}\left(u-\frac{d w}{d \phi}\right)\right]+\frac{1}{r_{1}^{2}}\left(u-\frac{d w}{d \phi}\right)^{2}\right.\right. \\
& \left.\left.+v \frac{\cot \phi}{r_{2}} \frac{1}{r_{1}}\left(u-\frac{d w}{d \phi}\right)\right]\right) \\
& -B \frac{r}{r_{1}} \cos \left[v\left(\frac{1}{r_{1}} \frac{d}{d \phi}\left[\frac{1}{r_{1}}\left(u-\frac{d w}{d \phi}\right)\right]+\frac{1}{r_{1}^{2}}\left(u-\frac{d w}{d \phi}\right)^{2}\right)\right. \\
& \left.+\frac{\cot \phi}{r_{2}} \frac{1}{r_{1}}\left(u-\frac{d w}{d \phi}\right)\right] \\
& -K r \vartheta\left[\frac{1}{r_{1}}\left(\frac{d u}{d \phi}+w\right)+\frac{1}{2} \vartheta^{2}+v \frac{1}{r_{2}}(u \cot \phi+w)\right] \\
& +r_{1} r f_{\phi}=0,
\end{aligned}
$$

$$
\begin{aligned}
& B \frac{d}{d \phi}\left[\frac { 1 } { r } \frac { d } { d \phi } \left[r \left[\frac{1}{r_{1}} \frac{d}{d \phi}\left[\frac{1}{r_{1}}\left(u-\frac{d w}{d \phi}\right)\right]+\frac{1}{r_{1}^{2}}\left(u-\frac{d w}{d \phi}\right)^{2}\right.\right.\right. \\
& \left.\left.\left.+v \frac{\cot \phi}{r_{2}} \frac{1}{r_{1}}\left(u-\frac{d w}{d \phi}\right)\right]\right]\right] \\
& -B \frac{d}{d \phi}\left[\operatorname { c o s } \phi \left[v\left(\frac{1}{r_{1}} \frac{d}{d \phi}\left[\frac{1}{r_{1}}\left(u-\frac{d w}{d \phi}\right)\right]+\frac{1}{r_{1}^{2}}\left(u-\frac{d w}{d \phi}\right)^{2}\right)\right.\right. \\
& \left.\left.+\frac{\cot \phi}{r_{2}} \frac{1}{r_{1}}\left(u-\frac{d w}{d \phi}\right)\right]\right] \\
& -K r\left[\frac{1}{r_{1}}\left(\frac{d u}{d \phi}+w\right)+\frac{1}{2} \vartheta^{2}+v \frac{1}{r_{2}}(u \cot \phi+w)\right] \\
& -K \frac{r_{1} r}{r_{2}}\left[\frac{1}{r_{2}}(u \cot \phi+w)+v \frac{1}{r_{1}}\left[\left(\frac{d u}{d \phi}+w\right)+\frac{1}{2} \vartheta^{2}\right]\right] \\
& -K \frac{d}{d \phi}\left(r \vartheta\left[\frac{1}{r_{1}}\left(\frac{d u}{d \phi}+w\right)+\frac{1}{2} \vartheta^{2}+v \frac{1}{r_{2}}(u \cot \phi+w)\right]\right) \\
& +r_{1} r f_{\zeta}=0 .
\end{aligned}
$$

Together, Eqs. 17 and 18 are an ODE system for $u, w$, taking into account of the principal radii $r_{1}, r_{2}$ in Eq. 7 and total rotation $\vartheta=\frac{1}{r_{1}}\left(u-\frac{d w}{d \phi}\right)$. Clearly the ODE system has no analytical solution and we must turn to numerics.

\section{Numerical study of the elastic oblique elliptic torus}

With the help of the code, we simulate three different tori: circular, normal and oblique elliptic tori. For simplification of presentation, physical units will not be plotted in all drawings, and they are listed in Table 1.
Table 1

Physical units used in this paper

\begin{tabular}{c|c|c|c|c|c|c}
\hline$R$ & $a, b$ & $h$ & $E$ & $v$ & $M_{\phi}$ & $N_{\phi}$ \\
\hline$m$ & $m$ & $m$ & $N / m^{2}$ & 1 & $N$ & $N / m$ \\
\hline \hline$Q_{\phi}$ & $\sigma_{\phi}$ & $u$ & $w$ & $\delta_{z}, \Delta_{z}$ & & \\
\hline$N / m$ & $N / m^{2}$ & $m$ & $m$ & $m$ & \multicolumn{2}{l}{} \\
\hline
\end{tabular}

\subsection{A circular torus}

One special case of elliptic torus is the circular torus with a cut along its parallel at $\phi=\frac{\pi}{2}$ or $\phi=-\frac{\pi}{2}$ under load $F$, as shown in Fig. 13.

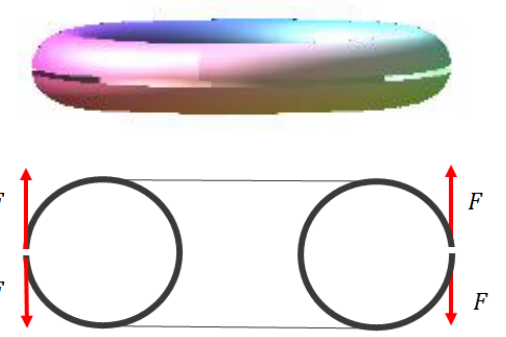

Figure 13: Torus with a cut along its parallel at $\phi=\frac{\pi}{2}$ or $\phi=-\frac{\pi}{2}$ acted by opposite and self-balance force $F=1[N]$. The boundary condition is: $\phi=\frac{\pi}{2}: N_{\phi}=-\frac{F}{2 \pi(R+a)}, Q_{\phi}=$ $0, M_{\phi}=0$ and $\phi=-\frac{3 \pi}{2}: N_{\phi}=-\frac{F}{2 \pi(R+a)}, Q_{\phi}=0, M_{\phi}=0$.

The numerical results of circular torus are shown in Fig. 14.

The bending movement, stress and displacement $w$ are strongly affected by the change of the ratio $\frac{a}{R}$, while the shear force and membrane force are much less affected.

\subsection{A normal elliptic torus}

When there were no oblique angle, namely $\beta=0$, all formulations reduced to the normal elliptic torus. Normal torus with a cut along its parallel at $\phi=\frac{\pi}{2}$ or $\phi=-\frac{\pi}{2}$ under load $F$, as shown Fig.15.

With the help of Maple, numerical results are obtained and shown in Fig. 16.

The numerical simulation show that the bending movement, stress and displacement $w$ are strongly affected by the change of the ratio $\frac{a}{R}$, while the shear force and membrane force are much less affected.

\subsection{Oblique elliptic torus vs. normal elliptic torus}

For a complete torus with a cut along the parallel $\theta=\frac{\pi}{2}$ and loaded with bending moment $M$, the loading condition is shown in Fig. 17.

From results shown in Fig. 18, it is clear that the rigidity can be achieved by adjusting the ratio of $a / b$ and oblique angle $\beta$.

For a complete torus with a cut along the parallel $\theta=\frac{\pi}{2}$ and loaded with opposite and self-balance force $F=1[N]^{2}$, the loading condition is shown in Fig. 19. 
Geometry-Induced Rigidity in Elastic Torus

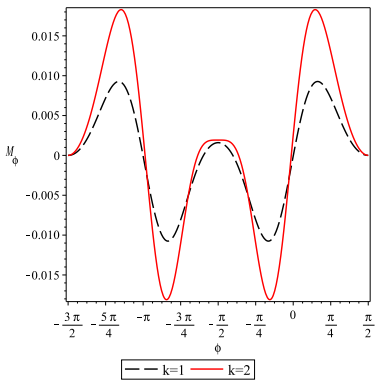

(a) Bending moment $M_{\phi}$

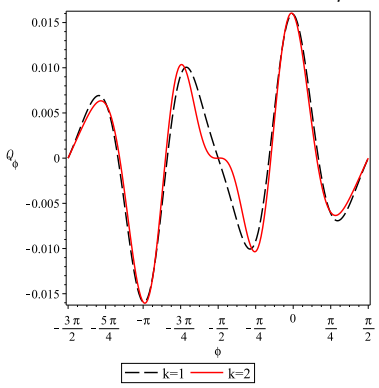

(c) Shear force $Q_{\phi}$

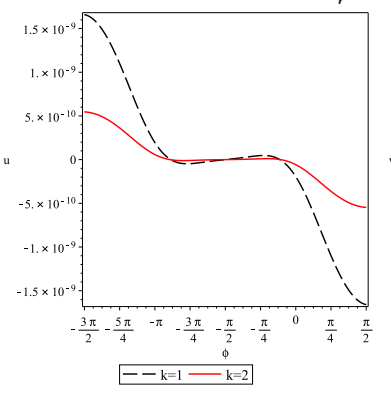

(e) Displacement $u$ in $\varphi$ direction,

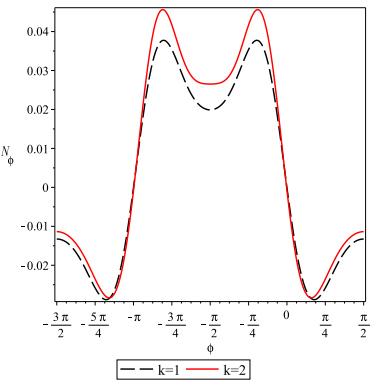

(b) Membranes force $N_{\phi}$

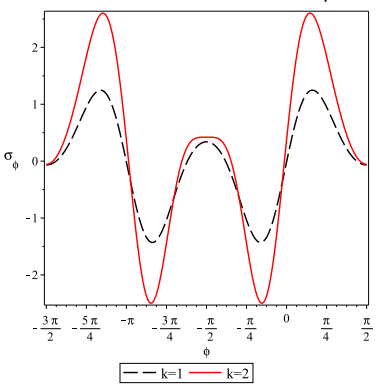

(d) Stress $\sigma_{\phi}$

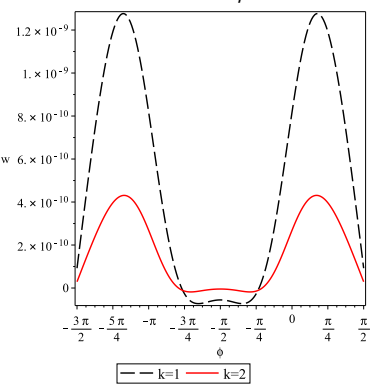

(f) Displacement $w$ in $\phi$ direction

Figure 14: Circular torus data: $a=b=2 k[m], R=$ $10[\mathrm{~m}], h=a / 20[\mathrm{~m}], \beta=0, E=2.0 \times 10^{11} \mathrm{~N} / \mathrm{m}^{2}, v=0.3, F=$ $1[N]$, where $k=1,2$

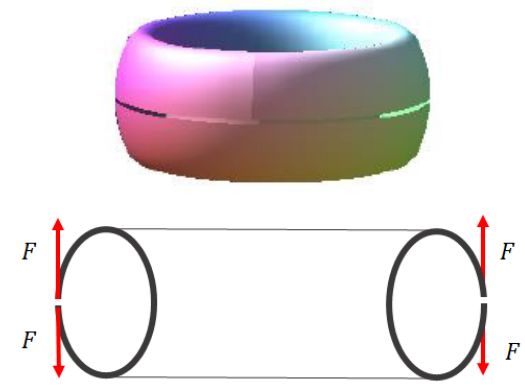

Figure 15: Normal torus with a cut along its parallel at $\phi=\frac{\pi}{2}$ or $\phi=-\frac{\pi}{2}$ acted by opposite and self-balance force $F=1[N]$. The boundary conditions: $\phi=\frac{\pi}{2}: N_{\phi}=-\frac{F}{2 \pi(R+a)}, Q_{\phi}=$ $0, M_{\phi}=0$ and $\phi=-\frac{3 \pi}{2}: N_{\phi}=-\frac{F}{2 \pi(R+a)}, Q_{\phi}=0, M_{\phi}=0$.

From results shown in Fig. 20, it is clear that the rigidity can be achieved by adjusting the ratio of $a / b$ and oblique angle $\beta$.

Similar to the circular and normal elliptic torus, the bending moment, shear force and membrane force, stress and de-

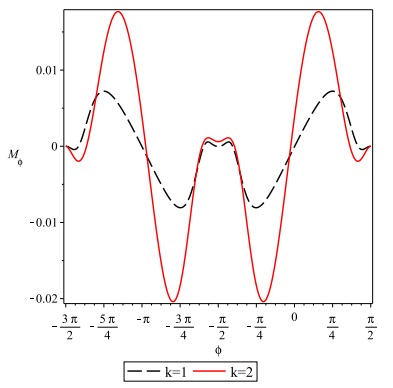

(a) Bending moment $M_{\phi}$

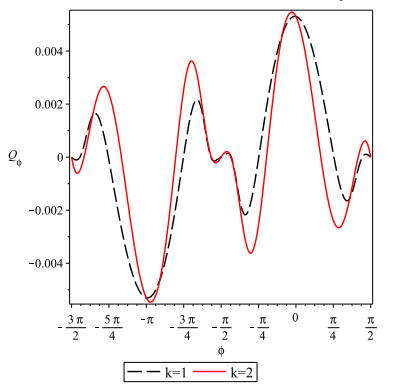

(c) Shear force $Q_{\phi}$
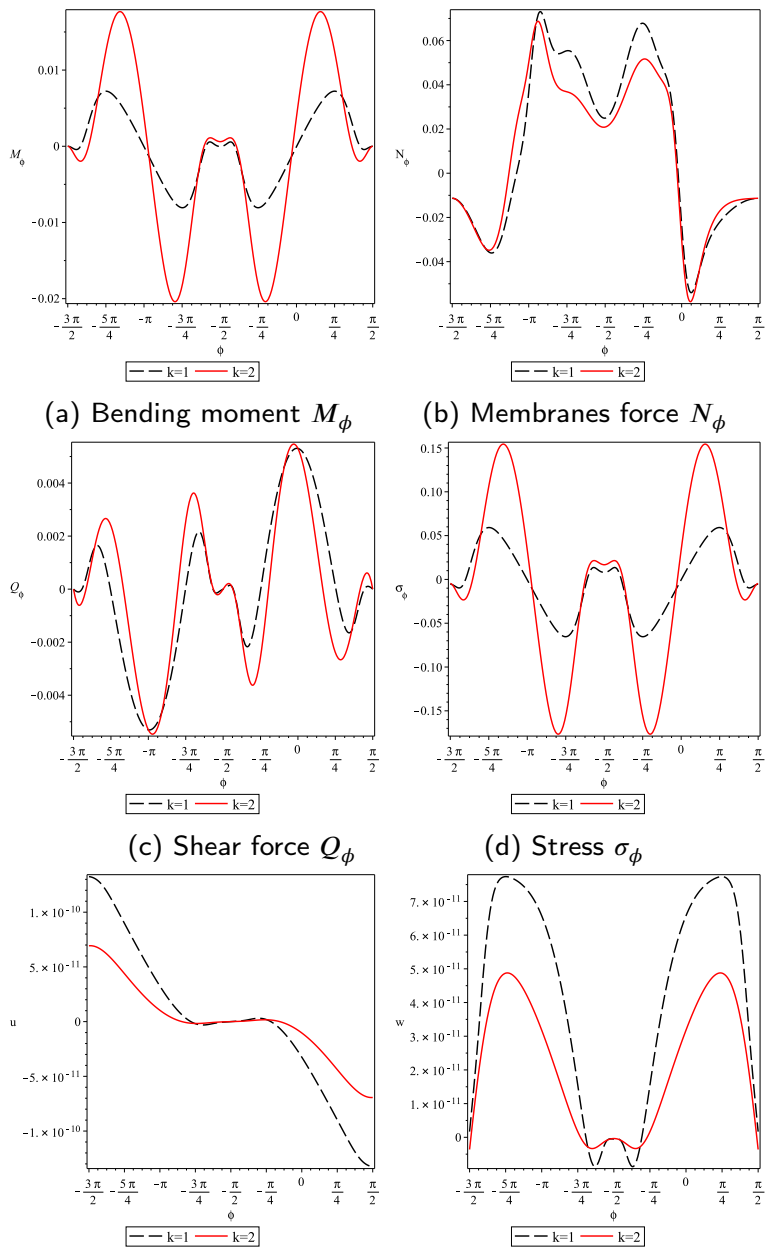

(b) Membranes force $N_{\phi}$

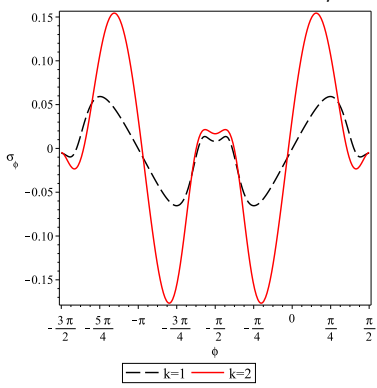

(d) Stress $\sigma_{\phi}$

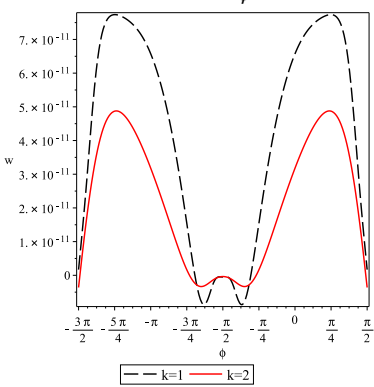

(e) Displacement $u$ in $\varphi$ direction, (f) Displacement $w$ in $\phi$ direction

Figure 16: Deformation caused vertical force. Normal elliptic torus with data: $a=6 k[\mathrm{~m}], b=15, R=30[\mathrm{~m}], h=$ $a / 15[\mathrm{~m}], \beta=0, E=2.0 \times 10^{11} \mathrm{~N} / \mathrm{m}^{2}, v=0.3, F=1[\mathrm{~N}]$, where $k=1,2$.

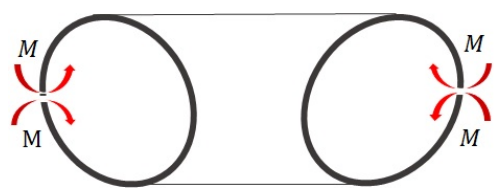

Figure 17: Torus with a cut along its parallel at $\phi=\frac{\pi}{2}$ or $\phi=-\frac{\pi}{2}$ under distributed bending moment $M=1[N] /[\mathrm{m}]$. The boundary conditions: $\phi=\frac{\pi}{2}: N_{\phi}=0, Q_{\phi}=0, M_{\phi}=1$ and $\phi=-\frac{3 \pi}{2}: N_{\phi}=0, Q_{\phi}=0, M_{\phi}=1$.

formation $w$ are strongly affected by the change of the angle $\beta$.

\subsection{Discussions}

The numerical results indicate that both deformation and stress response of an elastic torus are sensitive to the radius ratio, and all quantities such as bending moments, surface forces, shear force, and displacement are strongly affected by oblique angle. The study of Sun (2021) shows that analysis of a torus should be done by using the bending theory of 
Geometry-Induced Rigidity in Elastic Torus

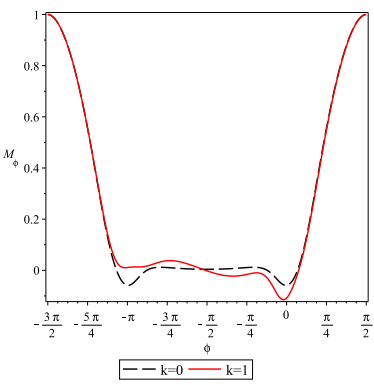

(a) Bending moment $M_{\phi}$

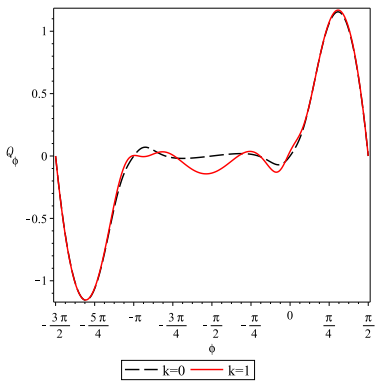

(c) Shear force $Q_{\phi}$

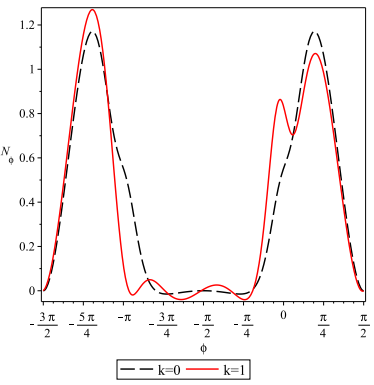

(b) Membranes force $N_{\phi}$

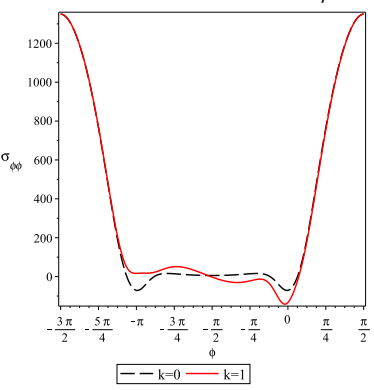

(d) Stress $\sigma_{\phi}$

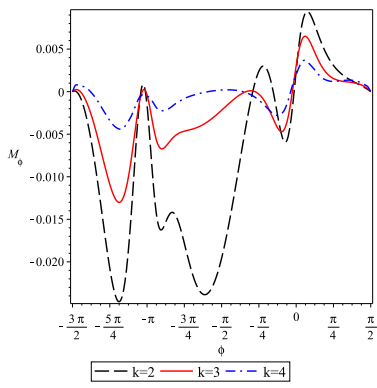

(a) Bending moment $M_{\phi}$

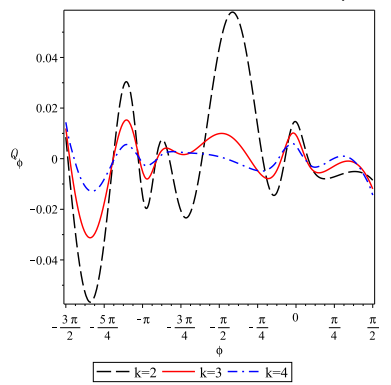

(c) Shear force $Q_{\phi}$

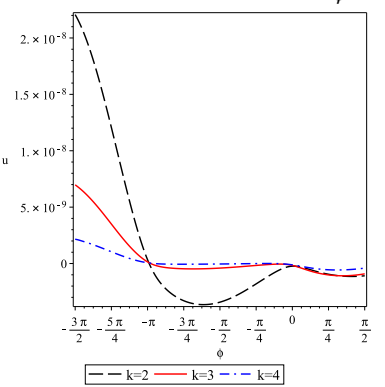

(c) Displacement $u$

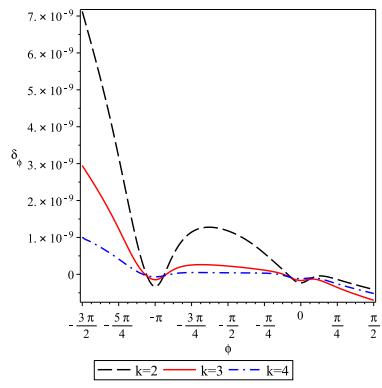

(c) Displacement in $x$

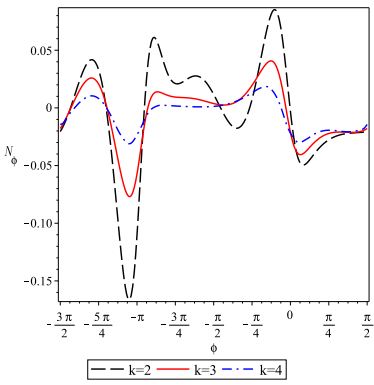

(b) Membranes force $N_{\phi}$

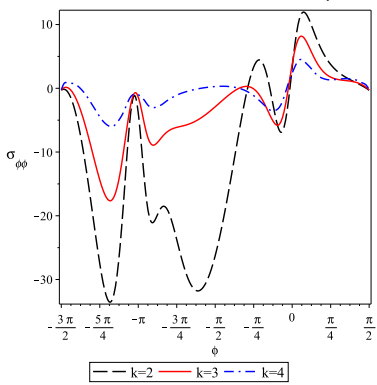

(d) Stress $\sigma_{\phi}$

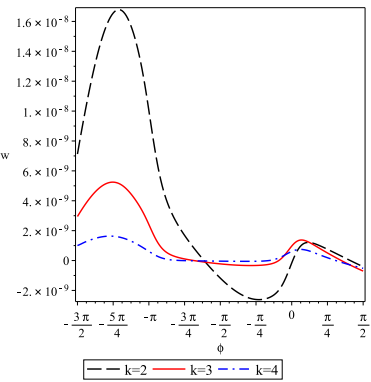

(d) Displacement $w$

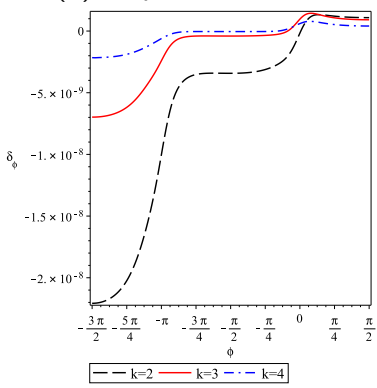

(d) Displacement in $z$

Figure 20: Oblique elliptic torus with data: $a=2[\mathrm{~m}], b=$ $1[\mathrm{~m}], R=5[\mathrm{~m}], h=b / 15[\mathrm{~m}], E=2.0 \times 10^{11} \mathrm{~N} / \mathrm{m}^{2}, v=$ $0.3, M=1[N]$ and $\beta=\frac{\pi}{16} k$, where $k=2,3,4$

ing term is not delectable due to the variation of curvature of the torus.

From the vertical displacement of the above-mentioned results, we can see that the vertical displacements are mainly contributed by the outer torus region and the region near the crowns at $\phi=-\pi$ and $\phi=0$. The inner region of the torus has little deformation since it has a displacement platform, which reveals that the inner torus is stiffer than the outer torus region due to its negative Gaussian curvature. This proved that an elastic torus deforms in a very specific manner, as the strain and stress concentration in two very narrow regions, namely around the top and bottom crowns. This nature of the torus is very useful for structural meta-materials design, where the stronger cell with negative Gaussian cur-

vature is often needed. If we wish to have a flexible torus, we can reduce the thickness around the crowns, and vise-versa.

\section{Conclusions}

We formulated the elastic oblique elliptic torus in terms of displacement. To verify our formulation, we wrote a computational code in Maple and carried out some numerical simulations. Our investigations show that both the deformation and the stress response of an elastic torus are sensitive to the radius ratio. Therefore the analysis of a torus should be 


\section{Geometry-Induced Rigidity in Elastic Torus}

done by the bending theory of a shell rather than by membrane theory of shells. Our numerical studies also reveal that the inner torus is stiffer than outer the torus due to the property of their Gaussian curvature. Last but not least, the oblique angle has a strong influence on the mechanics of the elliptic torus, and a small angle misalignment will significantly cause the change of deformation and strength distribution. Therefore, the oblique elliptic torus is worth to be studied.

\section{Acknowledgement:}

I dedicate this paper to my supervisor Prof. Dr.-Ing Wei Zhang ( former name is Wei Chang ), who was the first Chinese scholar to study the deformation of the torus. I thank my students: Mr Jie Wei, for preparation of Fig.7 (a,b) and Fig.12; Mr Wenqi Shao for drawing of Fig.2(a) and Mr Yong Sun for the preparation of Fig.6. I also wish to express my deep gratitude to the contributing editor Prof. Michel Destrade for his kind English proofreading and all anonymous reviewers for their high-level academic comments that helped me to enhance the quality of this paper.

\section{Data availability:}

The data that support the findings of this study are available from the author.

\section{Appendix: Maple code of nonlinear formulations of elastic torus}

To solve numerically, a general code is written in Maple.

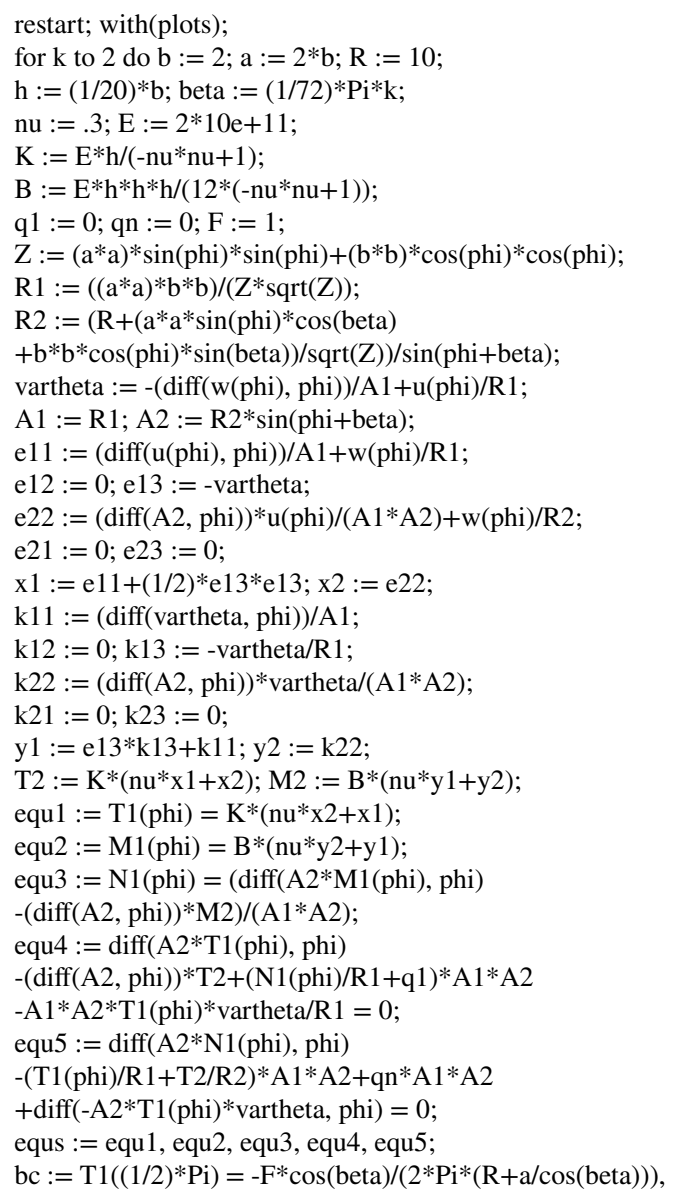

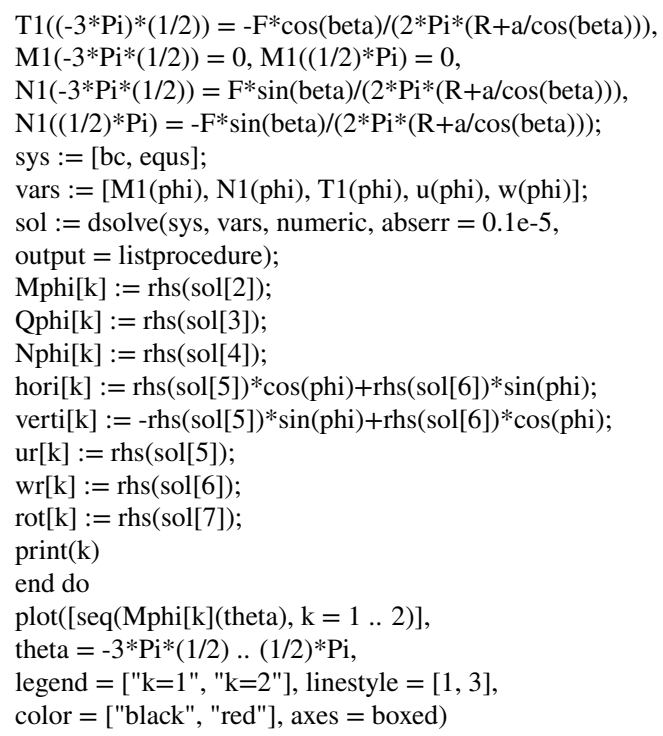

\section{References}

Audoly, B., Pomeau, Y., 2002. The elastic torus: anomalous stiffness of shells with mixed type. Comptes Rendus Mécanique. 330, 425-432.

Audoly, B., Pomeau, Y., 2010. Elasticity and Geometry. Oxford University Press.

Bielski, J., 1992. Influence of geometrical imperfections on buckling pressure and post-buckling behavior of elastic toroidal shells. Mechanics of Structures and Machines. 20, 145-154.

Calladine, C.R., 1983. Theory of Shell Structures. Cambridge University Press.

Chang, W., 1949. Der spannungszustand in kreisringschale und hnlichen schalen mit scheitelkreisringen unter drehsymmetrischer belastung (in german). Rep.Nat.Tsing Hua Univ., Ser. A. .

Clark, R.A., 1950. On the theory of thin elastic toroidal shells. Journal of Mathematics Physics. 29, 146-178.

Clark, R.A., Reissner, E., 1950. Bending of curved tubes. volume Advances in Applied Mechanics II. Academic Press.

Combescure, A., Galletly, G., 1999. Plastic buckling of complete toroidal shells of elliptical cross-section subjected to internal pressure. Thin Walled Structures. 34, 135-146.

Dong, M., 1985. New solution of novozhilov's equation of toroidal shells. Applied Mathematics and Mechanics. 6, 417-430.

Enoma, N., Zingoni, A., 2020a. Analytical formulation and numerical modelling for multi-shell toroidal pressure vessels. Computers and Structures. 232, 105811.

Enoma, N., Zingoni, A., 2020b. Buckling of an externally pressurised toroidal shell of revolution with a doubly-symmetric parabolic-ogival cross-section. International Journal of Pressure Vessels and Piping. 183, 104106.

Flügge, W., 1973. Stresses in shells. Springer Berlin Heidelberg .

Föppl, A., 1907. Vorlesungen über Technische Mechanik Vol 5. B.G.Teubner, Leipzig, Germany.

Galletly, G., 1998. Elastic buckling of complete toroidal shells of elliptical cross-section subjected to uniform internal pressure. Thin-walled Structures. 30, 23-34.

Galletly, G.D., Blachut, J., 1995. Stability of complete circular and noncircular toroidal shells. Proc. Inst. Mech. Engrs. 209(c), 245-255.

Gol'denveizer, A.L., 1961. Theory of Elastic Thin Shells. Pergamon Press, New York.

Jiammeepreecha, W., Chucheepsakul, S., 2017. Nonlinear static analysis of an underwater elastic semi-toroidal shell. Thin-Walled Structures. 116, $12-18$.

Jiammeepreecha, W., Chucheepsakul, S., 2019. Nonlinear free vibration analysis of a toroidal pressure vessel under constrained volume condition. Int. J. of Structural Stability and Dynamics. 19, 1950118. 


\section{Geometry-Induced Rigidity in Elastic Torus}

Kraus, H., 1967. The Elastic Shells. John Wileys \& Sons.

Kuznetsov, V.V., Levyakov, S.V., 2001. Nonlinear pure bending of toroidal shells of arbitrary cross-section. International Journal of Solids and Structures. 38, 7343-7354.

Lazarus, A., Florijn, H.C.B., Reis, P.M., 2012. Geometry-induced rigidity in nonspherical pressurized elastic shells. Physical Review Letters. 109, 144301.

Leung, A.Y., Kwok, N.T.C., 1994. Free vibration analysis of a toroidal shell. Thin-Walled Structures. 18, 317-332.

Libai, A., Simmonds, J., 1998. The Nonlinear Theory of Elastic Shells. Cambridge University Press.

Love, A.E.H., 1920. A Treatise on The Mathematical Theory of Elasticity, 3rd ed. Cambridge University Press.

Meissner, E., 1915. über und elastizitat festigkeit dünner schalen. Vierteljahrschrift der Naturforschungs, Gesellschaft, Zurich. 60, 23-47.

Ming, R.S., Pan, J., Norton, M.P., 2002. Free vibrations of elastic circular toroidal shells. Applied Acoustics. 63, 513-528.

Novozhilov, V.V., 1959. The Theory of Thin Shells. P. Noordhoff.

Reddy, J.N., 2007. Theory and Analysis of Elastic Plates and Shells. CRC Press.

Reissner, E., 1949. On bending of curved thin-walled tubes. Proceedings of the National Academy of Sciences of the United States of America. 35, 204-208.

Reissner, H., 1912. Spannungen in kugel-schalen (kuppeln). Festschrift Heinrich Müller-Breslau., 181-193.

Sanders, J.L.J., Liepins, A., 1969. Toroidal membrane under internal pressure. AIAA Journal. 1, 2105-2110.

Sobel, L., Függe, W., 1967. Stability of toroidal shells under uniform external pressure. AIAA Journal. 5, 425-431.

Steele, C.R., 1965. Toroidal pressure vessels. Journal of Spacecraft Rockets. 2, 937-943.

Sun, B.H., 2010. Closed form solution of axisymmetric slender elastic toroidal shells. ASCE Journal of Engineering Mechanics. 136, 12811288.

Sun, B.H., 2012. Toroidal Shells. Nova Publisher, New York.

Sun, B.H., 2013. Centenary studies of toroidal shells and in memory of prof. zhang wei (in chinese). Mechanics in Engineering. 37.

Sun, B.H., 2016. Exact solution of qian's equation of slender toroidal shells (in chinese). Mechanics in Engineering. 38, 567-569.

Sun, B.H., 2021. On symmetrical deformation of toroidal shell with circular cross-section. Thin-walled Structures. 163, 107680.

Sutcliffe, W.J., 1971. Stress analysis of toroidal shells of elliptical crosssection. International Journal of Mechanical Sciences. 13, 951-958.

Tao, L.N., 1959. On toroidal shells. Journal of Mathematics and Physics. $38,130-134$.

Timoshenko, S., Woinowsky-Krieger, S., 1959. Theory of Plates and Shells. Mcgraw-Hill College, New York.

Tölke, F., 1938. Zur integration der differentialgleichungen der drehsymmetrisch belasteten rotationsschale bei beliebiger wandstärke. IngenieurArchiv. 9, 282-288.

Vella, D., Ajdari, A., Vaziri, A., Boudaoud, A., 2012. Indentation of ellipsoidal and cylindrical elastic shells. Physical Review Letters. 109, 144302.

Venkadesan, M., Yawar, A., Eng, C.M., Dias, M.A., Singh, D.K., Tommasini, S.M., Haims, A.H., Bandi, M.M., Mandre, S., 2020. Stiffness of the human foot and evolution of the transverse arch. Nature. 579, 97-100.

Wissler, H., 1916. Festigkeitsberechnung von Ringflächen, Promotionarbeit. ETH, Zurich.

Xia, Z.H., Zhang, W., 1986. The general solution for thin-walled curved tubes with arbitrary loadings and various boundary conditions. International Journal of Pressure Vessels and Piping. 26, 129-144.

$\mathrm{Xu}$, B., Redekop, D., 2006. Natural frequencies of an orthotropic thin toroidal shell of elliptical cross-section. Journal of Sound and Vibration. 293, 440-448.

Yamada, G., Kobayashi, Y., Ohta, Y., Yokota, S., 1989. Free vibration of a toroidal shell with elliptical cross-section. Journal of Sound and Vibration. 135, 411-425.
Zhang, F., Redekop, D., 1992. Surface loading of a thin-walled toroidal shell. Computers and Structures. 43, 1019-1028.

Zhang, R.J., 1994. Toroidal shells under nonsymmetric loading. International Journal of Solids and Structures. 31, 2735-2750.

Zhang, R.J., Zhang, W., 1991. Turning point solutions for thin shell vibration. International Journal of Solids and Structures. 27, 1311-1326.

Zhang, W., Ren, W.M., Sun, B.H., 1990. Totoidal shells-history, current situation and future (in chinese). The 5th China National Conf. of Space Structures. (Lanzhou, China) .

Zingoni, A., 2018. Shell Structures in Civil and Mechanical Engineering. ICE Publishing.

Zingoni, A., Enoma, N., Govender, N., 2015. Equatorial bending of an elliptic toroidal shell. Thin-Walled Structures. 96, 286-294. 